\title{
Optimization Problems of Excess-of-Loss Reinsurance and Investment under the CEV Model
}

\author{
Qicai Li ${ }^{1,2}$ and Mengdi Gu${ }^{1}$ \\ ${ }^{1}$ Antai College of Economics and Management, Shanghai Jiao Tong University, Shanghai 200052, China \\ ${ }^{2}$ School of Mathematical Sciences, Nanjing Normal University, Nanjing 210046, China
}

Correspondence should be addressed to Qicai Li; liqicainj@gmail.com

Received 19 February 2013; Accepted 11 April 2013

Academic Editors: G. Gripenberg and G. Schimperna

Copyright (c) 2013 Q. Li and M. Gu. This is an open access article distributed under the Creative Commons Attribution License, which permits unrestricted use, distribution, and reproduction in any medium, provided the original work is properly cited.

\begin{abstract}
We consider that the insurer purchases excess-of-loss reinsurance and invests its wealth in the constant elasticity of variance (CEV) stock market. We model risk process by Brownian motion with drift and study the optimization problem of maximizing the exponential utility of terminal wealth under the controls of excess-of-loss reinsurance and investment. Using stochastic control theory and power transformation technique, we obtain explicit expressions for the optimal polices and value function. We also show that the optimal excess-of-loss reinsurance is always better than optimal proportional reinsurance. Some numerical examples are given.
\end{abstract}

\section{Introduction}

Many papers deal with optimal reinsurance or optimal investment issues for diffusion approximation risk models in the past ten years. In these papers, the insurer is allowed to take reinsurance and/or invest its capital in the BlackScholes market. Some of the problems have been dealt with through stochastic control theory and related methodologies for finding the minimum probability of ruin or the maximum expected utility of terminal wealth. Browne [1] used a Brownian motion with a drift to describe the surplus of the insurer and found the optimal investment policy to maximize the expected exponential utility of terminal wealth. Later, Schmidli [2], Taksar and Markussen [3] considered the optimal reinsurance policy which minimizes the ruin probability of the cedent.

Recently, much research on insurance optimization in the presence of both proportional reinsurance and investment has been done. Luo et al. [4] studied optimal proportional reinsurance and investment policy which minimizes the probability of ruin. Bai and Guo [5] investigated the problem of maximizing the expected exponential utility of terminal wealth with multiple risky assets and proportional reinsurance. For related works, see, for example, Promislow and Young [6], Liang and Guo [7] and references therein.
The excess-of-loss reinsurance has also attracted interest among academia and practitioners. Asmussen et al. [8] studied a dynamic choice of excess-of-loss reinsurance retention level and the dividend distribution policy which maximizes the expected present value of the dividends in a diffusion model. Zhang et al. [9] considered the problem of minimizing the probability of ruin by controlling the combinational quota-share and excess-of-loss reinsurance strategy. Meng and Zhang [10] investigated optimal risk control for the excess-of-loss reinsurance policy which minimizes the probability of ruin. Bai and Guo [11] explored optimal dynamic excess-of-loss reinsurance and multidimensional portfolio selection under the Black-Scholes market which maximizes the expected exponential utility of terminal wealth.

Although many papers are dealing with risk models with investment in the Black-Scholes market, there are analyses based on the other kinds of risk assets process in the actuarial literature. For example, Irgens and Paulsen [12] studied the optimal reinsurance and investment strategy with a jumpdiffusion process risk asset market. In fact, there is strong empirical evidence that the variance (or volatility) of asset returns, particularly stock market returns, is not constant [13]. The constant elasticity of variance $(\mathrm{CEV})$ process can describe stochastic volatility of the risky asset to some extent. The CEV 
model is expressed in terms of a stochastic diffusion process with respect to a standard Brownian motion:

$$
\mathrm{d} S(t)=\mu S(t) \mathrm{d} t+\sigma S(t)^{\beta+1} \mathrm{~d} W(t),
$$

where $\beta \leq 0$ is the elasticity parameter. This model is characterized by the dependence of the volatility rate, that is, $\sigma S(t)^{\beta+1}$ on the risk asset price. When the price increases, the instantaneous volatility rate decreases. This seems reasonable because the higher the stock price, the higher the equity market value, and thus the lower the proportion of liability, which results in a decrease in the risk of ruin. The volatility rate or the risk measure is thus decreased. So, the CEV model with stochastic volatility is a natural extension of the GBM (geometric Brownian motion) model. The CEV process was usually applied to calculating the theoretical price, sensitivities and implied volatility of option (see, e.g., Schroder [14]).

In this paper, we consider that the insurer purchases excess-of-loss reinsurance and invests its wealth in the CEV stock market. Although Gu et al. [15] used the CEV model to study the problems of reinsurance and investment under diffusion claim process, they get the optimal strategies for the proportional reinsurance. By the technique of stochastic control theory, we model risk process to Brownian motion with a drift to study the optimization problem of maximizing the exponential utility of terminal wealth under the controls of excess-of-loss reinsurance and investment, which is very different from those in $\mathrm{Gu}$ et al. The excess-of-loss reinsurance is a harder problem than proportional reinsurance from the mathematical point of view. Our contribution in this paper is to obtain explicit expressions for the optimal investment and excess-of-loss polices and value function. Moreover, we show that the excess-of-loss reinsurance dominates proportional reinsurance under our objective function. To the best of our knowledge, this is the first study to extend the research of $\mathrm{Gu}$ et al. to the case that the insurer purchases excess-of-loss reinsurance.

The rest of the paper is organized as follows. In Section 2, the model and assumption are given. In Section 3, we show that the optimal excess-of-loss reinsurance policy is always better than proportional reinsurance policy. The solution of the model is constructed in Section 4. In Section 5, some numerical examples are given.

\section{The Model and Assumptions}

2.1. Notation. Before introducing the mathematical models, some principal notations are listed.

$\{N(t), t \geq 0\}:$ the claim arrival process;

$Y_{i}$ : the claim size;

$c$ : the premium rate of the insurer without reinsurance policy;

$\eta, \theta$ : the safety loading of the insurer and reinsurer, respectively;

$a(t)$ : the retention level at time $t$;

$\pi(t)$ : the amount invested in the risky asset at time $t$; $\alpha=(\pi(t), a(t))$ : the admissible policy, denoted by $(\pi, a)$ for simplicity;

$c^{(a)}$ : the premium rate under the given reinsurance policy $a$;

$\tilde{Y}_{i}(a)$ : the part of the claim held by the insurer under the given reinsurance policy $a$;

$\mu(a), \sigma^{2}(a)$ : the first and second moments of $\widetilde{Y}_{i}(a)$, respectively;

$\left\{S_{0}(t), t \geq 0\right\},\{S(t), t \geq 0\}$ : the price process of the risk-free asset and risky asset, respectively;

$\{X(t), t \geq 0\}$ : the surplus process of the insurer;

$V(t, x, s)$ : the optimal value function at time $t$.

2.2. Problem Formulation. Let $(\Omega, \mathscr{F}, P)$ be a probability space with filtration $\left\{\mathscr{F}_{t}, t \geq 0\right\}$ containing all objects defined as follows. In the classical Cramer-Lundberg model, the reserve of an insurer at time $t$, denoted by $P(t)$, evolves over time as

$$
P(t)=x_{0}+c t-\sum_{i=1}^{N(t)} Y_{i}
$$

where $x_{0}$ is the initial level of reserve and $\{N(t), t \geq 0\}$ is a Poisson process with intensity $\lambda$. And $Y_{1}, Y_{2}, \ldots$, independent of $\{N(t)\}$, are i.i.d. random variables with common continuous distribution $F$ having finite first and second moments $\mu_{\infty}, \sigma_{\infty}^{2}$, respectively. The premium rate $c$ is assumed to be calculated via the expected principle, that is,

$$
c=(1+\eta) \lambda \mu_{\infty}
$$

where $\eta>0$ is the relative safety loading of the insurer.

We now consider a modification of the above CramerLundberg model that takes into account the presence of reinsurance. Let $a$ be a retention level and $\widetilde{Y}_{i}(a)$ denote the part of the claims held by the insurer. In other words, $Y_{i}-\widetilde{Y}_{i}(a)$ is the residual part of $Y_{i}$ that is covered by the reinsurer. Then, for a given reinsurance policy $a$, the corresponding reserve process is

$$
P^{(a)}(t)=x_{0}+c^{(a)} t-\sum_{i=1}^{N(t)} \widetilde{Y}_{i}(a),
$$

where premium rate is

$$
\begin{aligned}
c^{(a)} & =(1+\eta) \lambda \mu_{\infty}-(1+\theta) \lambda E\left[Y_{i}-\tilde{Y}_{i}(a)\right] \\
& =(1+\theta) E\left[\widetilde{Y}_{i}(a)\right] \lambda+(\eta-\theta) \lambda \mu_{\infty},
\end{aligned}
$$

where $\theta$ denotes the safety loading of the reinsurer, the reinsurer also used the expected value principle. In this paper, we consider noncheap reinsurance, that is, $\theta>\eta$, which is reasonable in actuarial practice. Otherwise, the insurer could 
reinsure the whole claims. According to Grandell [16], $P^{(a)}(t)$ can be approximated by the diffusion process $\left\{R^{(a)}(t), t \geq 0\right\}$ :

$$
\begin{aligned}
\mathrm{d} R^{(a)}(t)= & \lambda\left[\theta E\left[\widetilde{Y}_{i}(a)\right]+(\eta-\theta) \mu_{\infty}\right] \mathrm{d} t \\
& +\sqrt{\lambda E\left[\left(\widetilde{Y}_{i}(a)\right)^{2}\right]} \mathrm{d} W(t),
\end{aligned}
$$

where $W(t)$ is a standard Brownian motion adapted to $\mathscr{F}_{t}$.

For the excess-of-loss reinsurance with retention level $a$ (i.e., $\left.\widetilde{Y}_{i}(a)=\min \left(Y_{i}, a\right)=Y_{i} \wedge a\right)$,

$$
\begin{aligned}
\mu(a) & =E\left[Y_{i} \wedge a\right] \\
& =\int_{0}^{a} y \mathrm{~d} F(y)+a \bar{F}(a) \\
& =\int_{0}^{a} \bar{F}(y) \mathrm{d} y, \\
\sigma^{2}(a) & =E\left[\left(Y_{i} \wedge a\right)^{2}\right] \\
& =\int_{0}^{a} y^{2} \mathrm{~d} F(y)+a^{2} \bar{F}(a) \\
& =\int_{0}^{a} 2 y \bar{F}(y) \mathrm{d} y,
\end{aligned}
$$

where $\bar{F}(y)=P\left(Y_{i}>y\right)$. Without loss of generality, we assume that $\lambda=1$; then the corresponding diffusion approximation claim process (6) becomes

$$
\mathrm{d} R^{(a)}(t)=\left[\theta \mu(a(t))+(\eta-\theta) \mu_{\infty}\right] \mathrm{d} t+\sigma(a(t)) \mathrm{d} W(t) .
$$

We assumed that an insurer is allowed to invest its surplus in financial market consisting of a risk-free asset (bond or bank account) and a risky asset (stock or mutual fund). Specifically, the risk-free price process is given by

$$
\mathrm{d} S_{0}(t)=r S_{0}(t) \mathrm{d} t
$$

where $r>0$ is the risk-free interest rate.

As previously mentioned, the CEV model has advantages over the GBM model because of the stochastic volatility rate. We describe the risky asset price process by

$$
\mathrm{d} S(t)=\mu S(t) \mathrm{d} t+\sigma S(t)^{\beta+1} \mathrm{~d} B(t),
$$

where $\mu(>r)$ is an expected instantaneous rate of the risky asset and $\sigma S^{\beta+1}(t)$ is a standard instantaneous volatility. $B(t)$ is another $\mathscr{F}_{t}$-adapted standard Brownian and independent of the claim process.

Remark 1. If $\beta<0$, it can generate a distribution with heavy left tail. Empirical evidence supports the CEV model in the stock market (see, e.g., Schroder [14]). If $\beta>0$, the situation is unrealistic.

Let $\alpha=\{(\pi(t), a(t)), t \geq 0\}$, denoted by $(\pi, a)$ for simplicity, be any admissible control policy which is a twodimensional $\mathscr{F}_{t}$-adapted stochastic process, where $\pi$ represents the amount invested in the risky asset at time $t$, and
$0 \leq a(t) \leq \infty$ represents the excess-of-loss level at time $t$; the set of all admissible policies is denoted by $\Pi$.

The dynamics of resulting surplus process can be described as

$$
\begin{aligned}
\mathrm{d} X(t)= & \pi(t) \frac{\mathrm{d} S(t)}{S(t)} \\
& +(X(t)-\pi(t)) \frac{\mathrm{d} S_{0}(t)}{S_{0}(t)}+\mathrm{d} R^{(a)}(t) \\
= & {\left[r X(t)+(\mu-r) \pi(t)+\theta \mu(a(t))+(\eta-\theta) \mu_{\infty}\right] \mathrm{d} t } \\
& +\sigma \pi(t) S(t)^{\beta} \mathrm{d} B(t)+\sigma(a(t)) \mathrm{d} W(t) .
\end{aligned}
$$

Remark 2. In this paper, we assume that continuous trading is allowed and all assets are infinitely divisible. We allowed $\pi(t)<0$ and $\pi(t)>X(t)$, that means we allowed the insurer to short sell the risky asset and borrow money from a bank for investing in the risky asset.

We are interested in maximizing the utility of the cedent's terminal wealth, say at time $T$. Let $u(x)$ be the utility function with $u^{\prime}>0$ and $u^{\prime \prime}<0$. For $\alpha \in \Pi$, we define the return function as

$$
V^{\alpha}(t, x, s)=E[u(X(T)) \mid X(t)=x, S(t)=s] .
$$

The optimal value function is defined as

$$
V(t, x, s)=\sup _{\alpha \in \Pi} V^{\alpha}(t, x, s) .
$$

Our objective is finding an optimal policy $\alpha^{*} \in \Pi$

$$
V(t, x, s)=V^{\alpha^{*}}(t, x, s) .
$$

In the case of proportional reinsurance, an explicit solution to this problem was found by $\mathrm{Gu}$ et al. [15]. However, the excess-of-loss reinsurance is a harder problem than proportional reinsurance from the mathematical point of view: the functional relation between $\mu(a)$ and $\sigma(a)$ is much more complicated even for a relatively simple distribution $F$ such as the exponential or uniform.

Remark 3. A variety of utility functions are studied for investment and consumption strategies by an individual; see, for example, Karatzas [17] and references therein. We assume that the insurer is a closely-held corporation with risk aversion for reasonable utility analysis (see, Mayers and Smith [18], Loubergé and Watt [19]).

\section{The Gain of Excess-of-Loss Reinsurance}

In this section, we will show that the optimal excess-ofloss reinsurance policy is always better than proportional reinsurance policy. For the proportional reinsurance with retention level $a_{\mathrm{pr}}$ (i.e., $\widetilde{Y}_{i}\left(a_{\mathrm{pr}}\right)=a_{\mathrm{pr}} Y_{i}, 0<a_{\mathrm{pr}} \leq 1$ ),

$$
\begin{gathered}
\mu\left(a_{\mathrm{pr}}\right)=E\left[a_{\mathrm{pr}} Y_{i}\right]=a_{\mathrm{pr}} \mu_{\infty}, \\
\sigma^{2}\left(a_{\mathrm{pr}}\right)=E\left[\left(a_{\mathrm{pr}} Y_{i}\right)^{2}\right]=a_{\mathrm{pr}}^{2} \sigma_{\infty}^{2} .
\end{gathered}
$$


Then, the diffusion claim process (6) becomes

$$
\mathrm{d} R^{\left(a_{\mathrm{pr}}\right)}(t)=\left[\theta a_{\mathrm{pr}} \mu_{\infty}+(\eta-\theta) \mu_{\infty}\right] \mathrm{d} t+a_{\mathrm{pr}} \sigma_{\infty} \mathrm{d} W(t) .
$$

Lemma 4. Let $0<a_{\mathrm{pr}} \leq 1$ be a (fixed) retention level in proportional reinsurance model satisfying the condition

$$
\sigma^{2}(a)=E\left[\left(Y_{i} \wedge a\right)^{2}\right]=E\left[\left(a_{\mathrm{pr}} Y_{i}\right)^{2}\right]=a_{\mathrm{pr}}^{2} \sigma_{\infty}^{2}
$$

then

$$
\mu(a)=E\left[Y_{i} \wedge a\right] \geq a_{\mathrm{pr}} E\left[Y_{i}\right]=a_{\mathrm{pr}} \mu_{\infty} .
$$

Proof. The proof of the lemma can be found in Bai and Guo [11].

Theorem 5. For all $(t, x, s) \in[0, T] \times R \times R$, there exists policy $\alpha \in \Pi$, satisfying

$$
V^{\alpha}(t, x, s) \geq V_{\mathrm{pr}}^{a_{\mathrm{pr}}^{*}}(t, x, s),
$$

where $V^{\alpha}(t, x, s)$ is the value function for the excess-of-loss reinsurance model and $V^{a_{\mathrm{pr}}^{*}}(t, x, s)$ is the optimal value function for the proportional reinsurance model.

Proof. Let $\left(a_{\mathrm{pr}}^{*}, \pi^{*}\right)$ be the optimal feedback retention level and investment policy for the proportional reinsurance model (see $\mathrm{Gu}$ et al. [15]). The dynamics of the resulting surplus process (11) becomes

$$
\begin{aligned}
\mathrm{d} X(t)= & {\left[r X(t)+(\mu-r) \pi^{*}(t)+\theta a_{\mathrm{pr}}^{*} \mu_{\infty}+(\eta-\theta) \mu_{\infty}\right] \mathrm{d} t } \\
& +\sigma \pi^{*}(t) S(t)^{\beta} \mathrm{d} B(t)+a_{\mathrm{pr}}^{*} \sigma_{\infty} \mathrm{d} W(t) .
\end{aligned}
$$

Since $\sigma^{2}(a)$ is continuous function with respect to $a$ and $\sigma^{2}(\infty)=\int_{0}^{\infty} y^{2} \mathrm{~d} F(y)=\sigma_{\infty}^{2}$, we can choose a feedback control $\alpha=(\pi, a)$ in the excess-of-loss model in such a way that $\pi=\pi^{*}$ and $\sigma^{2}(a(t))=\int_{0}^{a(t)} y^{2} \mathrm{~d} F(y)=\left(a_{\mathrm{pr}}^{*} \sigma_{\infty}\right)^{2}$. From Lemma 4 , we have $\mu(a(t)) \geq a_{\mathrm{pr}}^{*} \mu_{\infty}$. Hence, with the same diffusion coefficient, the drift coefficient of excess-of-loss reinsurance model is bigger, which implies that $V^{\alpha}(t, x, s) \geq$ $V^{a_{\mathrm{pr}}^{*}}(t, x, s)$.

Remark 6. From the proof of Theorem 5, we can know that the preference for excess-of-loss reinsurance does not depend on utility function. We can also see that the result is true under our objective function in the case of cheap reinsurance (similar to Asmussen et al. [8]). Note that the premium is calculated by means of the expected value principle in the model. However, other premium principles are used, for example, the variance principle (see, Waters [20], Hesselager [21]), there may be different from the result of Theorem 5. In general this is a complicated matter. We leave this problem as an area for future research.

From now on, we only consider the excess-of-loss reinsurance model.

\section{Solution to the Model under Exponential Utility}

Suppose now that the insurer has exponential utility

$$
u(x)=\lambda_{0}-\frac{\gamma}{m} e^{-m x},
$$

where $\gamma>0$ and $m>0$. This utility has constant absolute risk aversion (CARA) parameter $m$. Such utility functions play a prominent role in insurance mathematics and actuarial practice, since they are the only utility functions under which the principle of "zero utility" gives a fair premium, that is, independent of the level of reserves of an insurer (see Gerber [22]).

We use the standard dynamic programming approach to solve the problem of maximizing expected exponential utility. We see that if the optimal value function $V$ and its partial derivatives $V_{t}, V_{x}, V_{x x}, V_{s}, V_{s s}$, and $V_{s x}$ are continuous on $[0, T) \times R \times R$, then $V$ satisfies the following Hamilton-JacobiBellman (HJB) equation:

$$
\sup _{\pi \in R, a \in[0, N]} \mathscr{A}^{\alpha} V(t, x, s)=0,
$$

with boundary condition

$$
V(T, x, s)=u(x)
$$

where

$$
\begin{aligned}
& \mathscr{A}^{\alpha} V(t, x, s) \\
& =V_{t}+\left[r x+(\mu-r) \pi+\theta \mu(a)+\mu_{\infty}(\eta-\theta)\right] V_{x} \\
& +\mu s V_{s}+\frac{1}{2}\left[\pi^{2} \sigma^{2} s^{2 \beta}+\sigma^{2}(a)\right] V_{x x} \\
& +\frac{1}{2} \sigma^{2} s^{2 \beta+2} V_{s s}+\pi \sigma^{2} s^{2 \beta+1} V_{x s}, \\
& N=\sup \{y: F(y)<1\} \leq \infty .
\end{aligned}
$$

The following verification theorem is essential in solving the associated stochastic control problem.

Theorem 7. Let $W \in C^{1,2}$ be concave solution to HJB equation (22) subject to the boundary condition (23). Then, the value function $V$ given by expression (13) coincides with $W$. That is,

$$
W(t, x, s)=V(t, x, s) .
$$

Furthermore, let $\left(\pi^{*}, a^{*}\right)$ be such that

$$
\mathscr{A}^{\left(\pi^{*}, a^{*}\right)} V(t, x, s)=0
$$

for all $(t, x, s) \in[0, T) \times R \times R$. Then, the feedback (Markov) strategies

$$
\left(\pi^{*}\left(t, X^{*}(t), S(t)\right), a^{*}\left(t, X^{*}(t), S(t)\right)\right)
$$

are the optimal policies.

Proof. The proof of the verification theorem is standard (see chapter III in Fleming and Soner [23]). 
Remark 8. In order to use the verification theorem in its basic form, a sufficient condition is that the following kind of expectation is finite:

$$
E \int_{t}^{t^{\prime}}\left|\mathscr{A}^{\left(\pi^{*}, a^{*}\right)} W\left(u, X^{*}(u), S(u)\right)\right| \mathrm{d} u<\infty, \quad \forall t^{\prime} \geq t,
$$

so as to be able to use Dynkin's formula and conclude that $W(t, x, s)$ is indeed $V(t, x, s)$. This point can be verified from the following conclusion of Theorem 9: $\pi^{*}(t)=O\left(1 / S(t)^{2 \beta}\right)$ and $a^{*}(t)=O(1)$

Theorem 9. When $N m>\theta$, the optimal value function is

$$
V(t, x, s)=\lambda_{0}-\frac{\gamma}{m} \exp \left[-m x e^{r(T-t)}+K_{1}(t)+L(t) s^{-2 \beta}\right],
$$

where

$$
\begin{gathered}
L(t)=-\frac{(\mu-r)^{2}}{4 r \sigma^{2}}\left(1-e^{-2 r(T-t)}\right), \\
K_{1}(t) \\
=\frac{\beta(2 \beta+1)(\mu-r)^{2}}{4 r}\left[(T-t)-\frac{1-e^{-2 r(T-t)}}{2 r}\right] \\
+\frac{m \mu_{\infty}(\eta-\theta) e^{r(T-t)}}{r} \int_{0}^{\theta e^{-r(T-z)} / m} \bar{F}(y) \mathrm{d} y \\
-\int_{t}^{T}\left[-\theta m e^{r(T-z)} \int_{0}^{2} \int^{2 r(T-z)} \int^{\theta e^{-r(T-z)} / m} y \bar{F}(y) \mathrm{d} y\right] \mathrm{d} z .
\end{gathered}
$$

In this case, the optimal excess-of-loss reinsurance and investment policy is

$$
\begin{aligned}
\pi^{*}(t)= & \frac{2 r(\mu-r)+\beta(\mu-r)^{2}\left(1-e^{-2 r(T-t)}\right)}{2 r \sigma^{2} s^{2 \beta}} \\
& \times \frac{e^{-r(T-t)}}{m}=O\left(\frac{1}{S(t)^{2 \beta}}\right), \\
& a^{*}(t)=\frac{\theta e^{-r(T-t)}}{m}=O(1) .
\end{aligned}
$$

When $N m \leq \theta$, the optimal value function is $V(t, x, s)=$

$$
\begin{array}{r}
\lambda_{0}-\frac{\gamma}{m} \exp \left[-m x e^{r(T-t)}+K_{1}(t)+L(t) s^{-2 \beta}+k\right], \\
0 \leq t<\bar{T}, \\
\lambda_{0}-\frac{\gamma}{m} \exp \left[-m x e^{r(T-t)}+K_{2}(t)+L(t) s^{-2 \beta}\right],
\end{array}
$$

$\bar{T} \leq t<T$, where $\bar{T}=T+(\ln (N m)-\ln \theta) / r$,

$$
\begin{aligned}
K_{2}(t)= & -\frac{\beta(2 \beta+1)(\mu-r)^{2}}{4 r}\left[(T-t)-\frac{1-e^{-2 r(T-t)}}{2 r}\right] \\
& -\frac{m \mu_{\infty} \eta}{r}\left(e^{r(T-t)}-1\right)-\frac{m \sigma_{\infty}^{2}}{4 r}\left(1-e^{2 r(T-t)}\right),
\end{aligned}
$$

and $k=K_{2}(\bar{T})-K_{1}(\bar{T})$. In this case, the corresponding optimal excess-of-loss reinsurance and investment policy is

$$
\left(\pi^{*}(t), a^{*}(t)\right)= \begin{cases}\left(\pi^{*}(t), \frac{\theta e^{-r(T-t)}}{m}\right), & 0 \leq t<\bar{T}, \\ \left(\pi^{*}(t), N\right), & \bar{T} \leq t<T,\end{cases}
$$

where $\pi^{*}(t)=\left(\left(2 r(\mu-r)+\beta(\mu-r)^{2}\left(1-e^{-2 r(T-t)}\right)\right) / 2 r \sigma^{2} s^{2 \beta}\right)$ $\left(e^{-r(T-t)} / m\right)=O\left(1 / S(t)^{2 \beta}\right)$.

Remark 10. From Theorem 9, we can see that when the total expected claims exceed the ratio of the reinsurer's safety loading to the coefficient of risk aversion, that is, $N>m / \theta$, the optimal excess-of-loss portfolio retention is the ratio of discounted reinsurer's safety loading to the coefficient of risk aversion. If $N \leq m / \theta$, the optimal excess-of-loss policy is no reinsurance when $t \geq \bar{T}$ and is also the ratio of discounted reinsurer's safety loading to the coefficient of risk aversion when $t \leq \bar{T}$.

Proof. Following the methods of Browne [1] or Liang et al. [24], we conjecture a solution of the form

$$
V(t, x, s)=\lambda_{0}-\frac{\gamma}{m} \exp \left[-m x e^{r(T-t)}+G(t, s)\right]
$$

where $G(t, s)$ is a suitable function to be determined. And the boundary condition $V(T, x, s)=u(x)$ implies that

$$
G(T, s)=0
$$

Let $G_{t}, G_{s}$, and $G_{s s}$ be the partial derivatives of $G(t, s)$. Note that

$$
\begin{gathered}
V_{t}=\left[V(t, x, s)-\lambda_{0}\right]\left[m x r e^{r(T-t)}+G_{t}\right], \\
V_{x}=\left[V(t, x, s)-\lambda_{0}\right]\left[-m e^{r(T-t)}\right], \\
V_{s}=\left[V(t, x, s)-\lambda_{0}\right]\left[G_{s}\right], \\
V_{x x}=\left[V(t, x, s)-\lambda_{0}\right]\left[m^{2} e^{2 r(T-t)}\right], \\
V_{s s}=\left[V(t, x, s)-\lambda_{0}\right]\left[G_{s}^{2}+G_{s s}\right], \\
V_{x s}=\left[V(t, x, s)-\lambda_{0}\right]\left[-m e^{r(T-t)} G_{s}\right] .
\end{gathered}
$$


Substituting (37) back into the HJB equation (22), since $V(t, x, s)-\lambda_{0}<0$, we get

$$
\begin{aligned}
G_{t}- & m \mu_{\infty}(\eta-\theta) e^{r(T-t)}+\mu s G_{s}+\frac{1}{2} \sigma^{2} s^{2 \beta+2}\left(G_{s}^{2}+G_{s s}\right) \\
+ & \inf _{\pi \in R}\left\{\left[-(\mu-r) \pi-\pi \sigma^{2} s^{2 \beta+1} G_{s}\right.\right. \\
& \left.\left.+\frac{1}{2} \sigma^{2} \pi^{2} s^{2 \beta} m e^{r(T-t)}\right] m e^{r(T-t)}\right\} \\
+ & \inf _{a \in[0, N]}\left\{\left[-\mu(a) \theta+\frac{1}{2} \sigma^{2}(a) m e^{r(T-t)}\right] m e^{r(T-t)}\right\}=0 .
\end{aligned}
$$

Let

$$
\begin{gathered}
f_{1}(\pi, t) \\
=\left[-(\mu-r) \pi-\pi \sigma^{2} s^{2 \beta+1} G_{s}+\frac{1}{2} \sigma^{2} \pi^{2} s^{2 \beta} m e^{r(T-t)}\right] m e^{r(T-t)}, \\
f_{2}(a, t)=\left[-\mu(a) \theta+\frac{1}{2} \sigma^{2}(a) m e^{r(T-t)}\right] m e^{r(T-t)} .
\end{gathered}
$$

Differentiating $f_{1}(\pi, t)$ with respect to $\pi$ yields the minimizer

$$
\pi^{*}=\frac{(\mu-r)+\sigma^{2} s^{2 \beta+1} G_{s}}{\sigma^{2} s^{2 \beta}} \frac{e^{-r(T-t)}}{m},
$$

and the value of $f_{1}(\pi, t)$ at this minimizer is

$$
f_{1}\left(\pi^{*}, t\right)=-\frac{1}{2} \frac{\left[(\mu-r)+\sigma^{2} s^{2 \beta+1} G_{s}\right]^{2}}{\sigma^{2} s^{2 \beta}} .
$$

Similarly, from the first order condition

$$
\frac{\partial f_{2}(a, t)}{\partial a}=\left[-\theta \bar{F}(a)+a \bar{F}(a) m e^{r(T-t)}\right] m e^{r(T-t)}=0,
$$

we know that without restriction with respect to $a$,

$$
\tilde{a}=\frac{\theta}{m} e^{-r(T-t)},
$$

which leads to

$$
\begin{aligned}
f_{2}(\widetilde{a}, t)= & {\left[-\mu(\widetilde{a}) \theta+\frac{1}{2} \sigma^{2}(\widetilde{a}) m e^{r(T-t)}\right] m e^{r(T-t)} } \\
= & -\theta m e^{r(T-t)} \int_{0}^{\theta e^{-r(T-t)} / m} \bar{F}(y) \mathrm{d} y \\
& +m^{2} e^{2 r(T-t)} \int_{0}^{\theta e^{-r(T-t)} / m} y \bar{F}(y) \mathrm{d} y .
\end{aligned}
$$

We need to discuss the two cases according to the value of $\widetilde{a}$.

Case 1. When $N m>\theta$

$$
\begin{gathered}
\text { if } t<T+(\ln (N m)-\ln \theta) / r \text {, then } \tilde{a} \in[0, N) \text {. So, } \\
\left(\pi^{*}(t), a^{*}(t)\right)=\left(\frac{(\mu-r)+\sigma^{2} s^{2 \beta+1} G_{s}}{\sigma^{2} s^{2 \beta}} \cdot \frac{e^{-r(T-t)}}{m}, \frac{\theta e^{-r(T-t)}}{m}\right)
\end{gathered}
$$

coincides with the optimal policy. Since $T<T+(\ln (N m)-$ $\ln \theta) / r,\left(\pi^{*}(t), a^{*}(t)\right)$ is optimal policy on $[0, T]$.

Up to now, we still need to solve $G(t, s)$ to find $\pi^{*}(t)$ and $V(t, x, s)$ in this case. Substituting $\left(\pi^{*}(t), a^{*}(t)\right)$ (i.e., expression (45)) back to (38), we can get

$$
\begin{aligned}
G_{t}- & m \mu_{\infty}(\eta-\theta) e^{r(T-t)}+r s G_{s}+\frac{1}{2} \sigma^{2} s^{2 \beta+2} G_{s s} \\
- & \frac{(\mu-r)^{2}}{2 \sigma^{2} s^{2 \beta}}+f_{2}\left(a^{*}, t\right)=0 .
\end{aligned}
$$

We appeal to power transformation technique and variable change method to solve the problem.

Let

$$
G(t, s)=h(t, y), \quad y=s^{-2 \beta},
$$

with boundary condition

$$
\begin{gathered}
h(T, y)=0, \\
G_{t}=h_{t}, \quad G_{s}=-2 \beta s^{-2 \beta-1} h_{y}, \\
G_{s s}=2 \beta(2 \beta+1) s^{-2 \beta-2} h_{y}+4 \beta^{2} s^{-4 \beta-2} h_{y y},
\end{gathered}
$$

where $h_{t}, h_{y}$, and $h_{y y}$ are partial derivatives of $h(t, y)$.

Putting the partial derivatives of $G(t, s)$ into (46), we obtain

$$
\begin{aligned}
h_{t}+ & {\left[\sigma^{2}(2 \beta+1)-2 r y\right] \beta h_{y}+2 \sigma^{2} \beta^{2} y h_{y y} } \\
& -\frac{(\mu-r)^{2}}{2 \sigma^{2}} y+M_{1}(t)=0,
\end{aligned}
$$

where $M_{1}(t)=-m \mu_{\infty}(\eta-\theta) e^{r(T-t)}+f_{2}\left(a^{*}, t\right)$. We try to find a solution of the above equation with the following form

$$
h(t, y)=K_{1}(t)+L_{1}(t) y \text {, }
$$

with boundary condition

$$
\begin{gathered}
K_{1}(T)=0, \\
L_{1}(T)=0, \\
h_{t}=K_{1}^{\prime}+L_{1}^{\prime} y, \quad h_{y}=L_{1}, \quad h_{y y}=0,
\end{gathered}
$$

where $K_{1}^{\prime}, L_{1}^{\prime}$ are the derivatives of $K_{1}, L_{1}$, respectively. Putting (53) into (49), we derive

$$
\begin{aligned}
& K_{1}^{\prime}+\sigma^{2} \beta(2 \beta+1) L_{1}+\left[L_{1}^{\prime}-2 r L_{1}-\frac{(\mu-r)^{2}}{2 \sigma^{2}}\right] y \\
& \quad+M_{1}(t)=0 .
\end{aligned}
$$

By matching coefficients, we have

$$
\begin{gathered}
K_{1}^{\prime}+\sigma^{2} \beta(2 \beta+1) L_{1}+M_{1}(t)=0, \\
L_{1}^{\prime}-2 r L_{1}-\frac{(\mu-r)^{2}}{2 \sigma^{2}}=0 .
\end{gathered}
$$


Taking into account the boundary condition, we have the solution of (55):

$$
\begin{gathered}
L_{1}(t)=-\frac{(\mu-r)^{2}}{4 r \sigma^{2}}\left(1-e^{-2 r(T-t)}\right), \\
K_{1}(t)=\int_{t}^{T}\left[\beta(2 \beta+1) \sigma^{2} L_{1}(z)+M_{1}(z)\right] \mathrm{d} z \\
=-\frac{\beta(2 \beta+1)(\mu-r)^{2}}{4 r}\left[(T-t)-\frac{1-e^{-2 r(T-t)}}{2 r}\right] \\
+\frac{m \mu_{\infty}(\eta-\theta)\left(e^{r(T-t)}-1\right)}{r} \\
+\int_{t}^{T}\left[-\theta m e^{r(T-z)}\left(\int_{0}^{\theta e^{-r(T-z)} / m} \bar{F}(y) \mathrm{d} y\right)\right. \\
\left.+m^{2} e^{2 r(T-z)}\left(\int_{0}^{\theta e^{-r(T-z)} / m} y \bar{F}(y) \mathrm{d} y\right)\right] \mathrm{d} z .
\end{gathered}
$$

Putting these parameters into $G(t, s)$, we obtain

$$
G(t, s)=K_{1}(t)+L_{1}(t) s^{-2 \beta}
$$

So, the optimal investment policy is

$$
\frac{2 r(\mu-r)+\beta(\mu-r)^{2}\left(1-e^{-2 r(T-t)}\right)}{2 r \sigma^{2} s^{2 \beta}} \cdot \frac{e^{-r(T-t)}}{m},
$$

and the corresponding value function has the form

$$
V(t, x, s)=\lambda_{0}-\frac{\gamma}{m} \exp \left[-m x e^{r(T-t)}+K_{1}(t)+L_{1}(t) s^{-2 \beta}\right],
$$

where $L_{1}(t)$ and $K_{1}(t)$ are determined by (56), respectively.

\section{Case 2. When $N m \leq \theta$}

If $t<T+(\ln (N m)-\ln \theta) / r$ (noting that $T+(\ln (N m)-$ $\ln \theta) / r \leq T)$, we know that $\tilde{a} \in[0, N)$ from expression (43). Similar to Case 1, incorporating the constants of the calculations, we get the optimal value function

$$
\begin{aligned}
& V(t, x, s) \\
& =\lambda_{0}-\frac{\gamma}{m} \exp \left[-m x e^{r(T-t)}+K_{1}(t)+L_{1}(t) s^{-2 \beta}+k\right],
\end{aligned}
$$

where the constant $k$ will be determined from the following (70), and the optimal policies are

$$
\left(\pi^{*}(t), a^{*}(t)\right)=\left(\frac{(\mu-r)+\sigma^{2} s^{2 \beta+1} G_{s}}{\sigma^{2} s^{2 \beta}} \frac{e^{-r(T-t)}}{m}, \frac{\theta e^{-r(T-t)}}{m}\right),
$$

where $G(t, s)=K_{1}(t)+L_{1}(t) s^{-2 \beta}+k$. If $T+(\ln (N m)-\ln \theta) / r \leq$ $t \leq T$, then $\tilde{a} \geq N$. We get that the optimal retention level is
$a^{*}(t)=N$. In this case, $\mu(N)=\mu_{\infty}$ and $\sigma^{2}(N)=\sigma_{\infty}^{2}$. Putting the optimal policies

$$
\left(\pi^{*}(t), a^{*}(t)\right)=\left(\frac{(\mu-r)+\sigma^{2} s^{2 \beta+1} G_{s}}{\sigma^{2} s^{2 \beta}} \frac{e^{-r(T-t)}}{m}, N\right),
$$

into (38), we obtain

$$
\begin{gathered}
G_{t}-m \mu_{\infty} \eta e^{r(T-t)}+r s G_{s}+\frac{1}{2} \sigma^{2} s^{2 \beta+2} G_{s s} \\
-\frac{1}{2} \frac{(\mu-r)^{2}}{\sigma^{2} s^{2 \beta}}+\frac{1}{2} \sigma_{\infty}^{2} m^{2} e^{2 r(T-t)}=0 .
\end{gathered}
$$

Again, we use the power transformation technique and variable change method to solve (63) with the boundary condition (21).

Similarly, let $G(t, s)=h(t, y), y=s^{-2 \beta}$, we have

$$
\begin{aligned}
h_{t} & +\left[\sigma^{2}(2 \beta+1)-2 r y\right] \beta h_{y}+2 \sigma^{2} \beta^{2} y h_{y y} \\
& -\frac{(\mu-r)^{2}}{2 \sigma^{2}} y-m \mu_{\infty} \eta e^{r(T-t)}+\frac{1}{2} \sigma_{\infty}^{2} m^{2} e^{2 r(T-t)}=0 .
\end{aligned}
$$

And we try the following form and match coefficients,

$$
h(t, y)=K_{2}(t)+L_{2}(t) y .
$$

Therefore, we get

$$
L_{2}(t)=-\frac{(\mu-r)^{2}}{4 r \sigma^{2}}\left(1-e^{-2 r(T-t)}\right)
$$

which is the same as the expression $L_{1}(t)$, denoted by $L(t)$ for simplicity:

$$
\begin{gathered}
K_{2}(t)=-\frac{\beta(2 \beta+1)(\mu-r)^{2}}{4 r}\left[(T-t)-\frac{1-e^{-2 r(T-t)}}{2 r}\right] \\
-\frac{m \mu_{\infty} \eta}{r}\left(e^{r(T-t)}-1\right)-\frac{m \sigma_{\infty}^{2}}{4 r}\left(1-e^{2 r(T-t)}\right), \\
G(t, s)=K_{2}(t)+L_{2}(t) s^{-2 \beta} .
\end{gathered}
$$

Let $\bar{T}=T+(\ln (N m)-\ln \theta) / r$. So, in this case, the optimal excess-of-loss reinsurance and investment policies are

$$
\left(\pi^{*}(t), a^{*}(t)\right)= \begin{cases}\left(\pi^{*}(t), \frac{\theta e^{-r(T-t)}}{m}\right), & 0 \leq t<\bar{T}, \\ \left(\pi^{*}(t), N\right), & \bar{T} \leq t<T,\end{cases}
$$

where $\pi^{*}(t)=\left(\left(2 r(\mu-r)+\beta(\mu-r)^{2}\left(1-e^{-2 r(T-t)}\right)\right) / 2 r \sigma^{2} s^{2 \beta}\right)$. $\left(e^{-r(T-t)} / m\right)$. And the corresponding value function has the form $V(t, x, s)=$

$$
\begin{array}{r}
\lambda_{0}-\frac{\gamma}{m} \exp \left[-m x e^{r(T-t)}+K_{1}(t)+L(t) s^{-2 \beta}+k\right], \\
0 \leq t<\bar{T}, \\
\lambda_{0}-\frac{\gamma}{m} \exp \left[-m x e^{r(T-t)}+K_{2}(t)+L(t) s^{-2 \beta}\right],
\end{array}
$$

$$
\bar{T} \leq t<T
$$




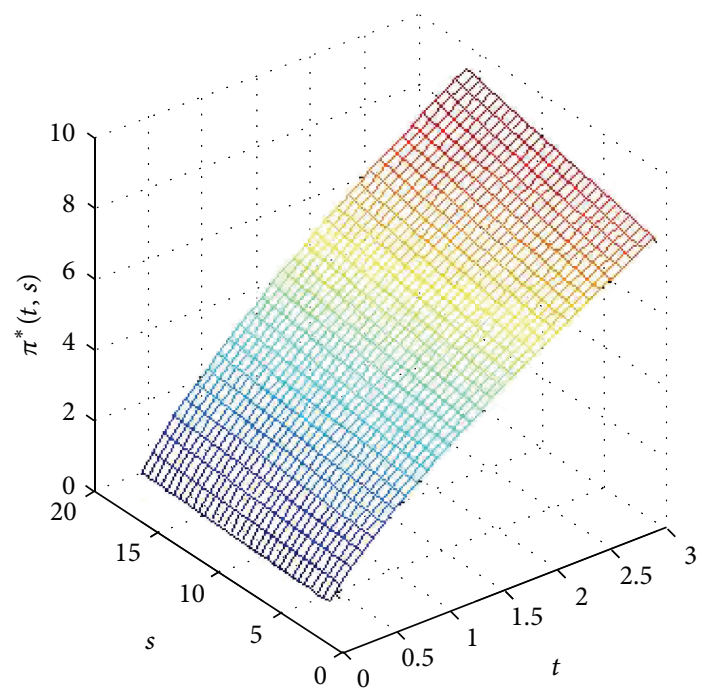

Figure 1: The surface of $\pi^{*}(t, s)$ with $m=0.1, \sigma=0.19, r=0.04$, $\mu=0.08$, and $\beta=-1 / 3$.

where choose $k$ in the way that $V(t, x, s)$ given by (69) is continuous at $\bar{T}$; that is,

$$
k=K_{2}(\bar{T})-K_{1}(\bar{T})
$$

Thus, we complete the proof.

Remark 11. From Theorem 9, we can see that the optimal investment policy is independent of claim size distribution $F$ and the value of $x$ but is dependent on the value of the risk asset price $s$ and time $t$.

\section{Numerical Examples of the Optimal Policies}

In this section, to give some intuitive interpretation of optimal investment and reinsurance policies, we demonstrate numerical examples of two main claim sizes distributionsthe exponential and uniform distributions. We set the riskless rate at $r=0.04$ per year, the mean excess returns at $\mu-r=$ 0.04 per year, and the parameter $\sigma$ in the expression of an annual standard volatility at 0.19 . The estimates of parameters can be based on annual equity return on the stock price index. We refer the readers to Chacko and Viceira [13] and Schroder [14] and references therein.

Let the time horizon $T=3$ years be fixed. Because the optimal investment policy is independent of claim size distribution, we firstly give the graph of the optimal investment policies in Figure 1 with the risk aversion parameter $m=0.1$ and the elasticity parameter $\beta=-1 / 3$.

From Figure 1, we can see that the effect of the risky asset price on the optimal investment policies $\pi^{*}(t, s)$ is relatively small. In practice, the optimal investment policy is comparatively more responsive to changes of the mean excess return and volatility of returns. We provide some reports concerning the sensitivity to these parameterizations in Figures 2 and 3.

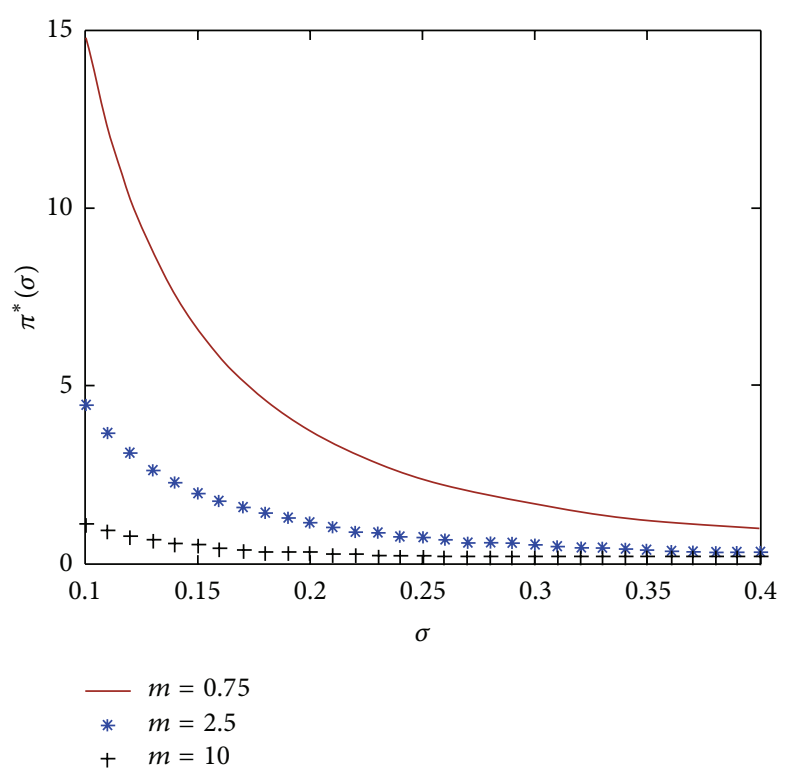

Figure 2: The effect of $\sigma$ on $\pi^{*}(2,5)$ with $\beta=-1 / 3$.

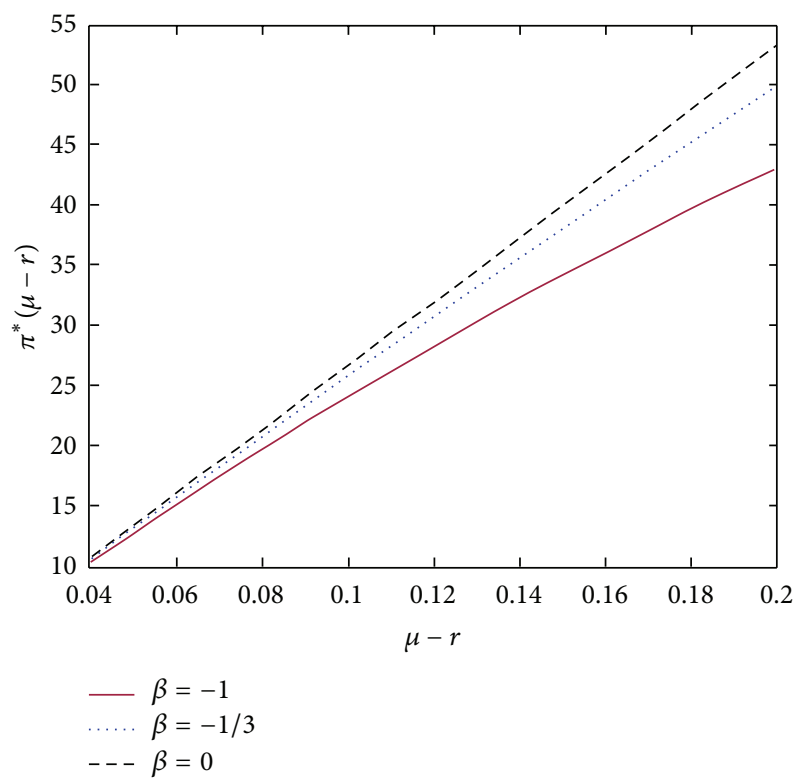

FIgURE 3: The effect of $\mu-r$ on $\pi^{*}(2,5)$ with $m=2.5$.

Let time $t=2$ and the risky asset price $s=5$ are fixed. We consider values of $\sigma$ between 0.1 and $0.4, \mu-r=0.04$, and $\beta=-1 / 3$ in Figure 2, and values of $\mu-r$ between 0.04 and $0.2, \sigma=0.19$, and $m=2.5$ in Figure 3 .

Figure 2 shows that the optimal investment policy decreases as the standard volatility increases. Moreover, a higher level $m$ yields a lower value of the optimal investment policy, which is the natural consequence since the larger value of $m$ means more risk aversion.

Figure 3 reports that the optimal investment policy increases as the excess return $\mu-r$ increases. The result also shows that a higher elasticity parameter $\beta$ yields a larger value 


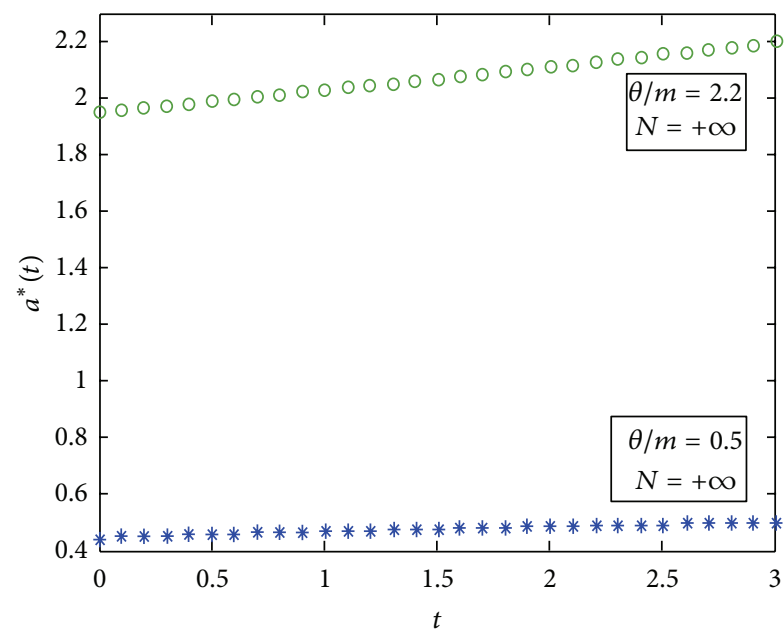

FIGURE 4: $Y \sim e(1)$, the optimal excess-of-loss reinsurance level $a^{*}(t)$ with $\theta / m=0.5,2.2$, respectively.

of the optimal investment policy. Especially, when $\beta$ attains its maximum 0 , the model degenerates to a GBM model.

The following examples are about reinsurance policy.

Example 1. Assume that the claim size is a standard exponential distribution, $\bar{F}(y)=e^{-y}$, then $N=+\infty$. In this case, $\mathrm{Nm}>\theta$ is always true. Thus, the optimal excess-ofloss reinsurance level $a^{*}(t)=(\theta / m) e^{-r(T-t)}$ on $[0, T]$. Figure 4 presents the optimal excess-of-loss reinsurance level for different $\theta / m=0.5,2.2$, respectively.

Example 2. Assume that the claim size is a uniform distribution on $(0,2)$, then $N=2$. If $\theta / m=0.5$, then $N m>\theta$. Thus, $a^{*}(t)=(\theta / m) e^{-r(T-t)}$ on $[0, T]$. If $\theta / m=2.2$, then $N m<\theta$. Thus, $a^{*}(t)=(\theta / m) e^{-r(T-t)}$ on $[0,0.617)$ and $a^{*}(t)=$ 2 on $[0.617,3]$. Figure 5 presents the optimal excess-of-loss reinsurance level for different $\theta / m=0.5,2.2$, respectively.

From Figure 5, we can see that if $\theta / m=2.2$, the optimal retention $a^{*}(t)$ is a linear increasing function with respect to $t$ when $t \in[0,0.617)$ and is flat for all $t \geq 0.617$. But, in Figure 4, the optimal retention $a^{*}(t)$ is always a linear increasing function with respect to $t$ since the potential maximal value of the claim size $Y_{i}$ is infinity.

\section{Acknowledgments}

This research was supported by SJTU Special Funds for Cross Disciplines (Grant no. 10JCY11) and the National Natural Science Foundation of China (Grant no. 11101215).

\section{References}

[1] S. Browne, "Optimal investment policies for a firm with random risk process: exponential utility and minimizing the probability of ruin," Mathematics of Operations Research, vol. 20, no. 4, pp. 937-958, 1995.

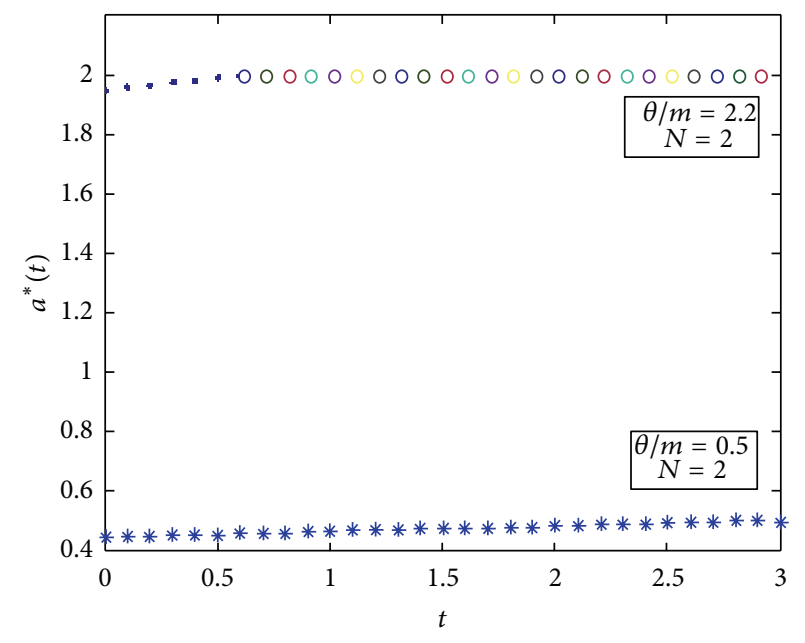

FIgURE 5: $Y \sim U(0,2)$, the optimal excess-of-loss reinsurance level $a^{*}(t)$ with $\theta / m=0.5,2.2$, respectively.

[2] H. Schmidli, "Optimal proportional reinsurance policies in a dynamic setting," Scandinavian Actuarial Journal, vol. 1, pp. 5568, 2001.

[3] M. Taksar and C. Markussen, "Optimal dynamic reinsurance policies for large insurance portfolios," Finance and Stochastics, vol. 7, pp. 97-121, 2003.

[4] S. Luo, M. Taksar, and A. Tsoi, "On reinsurance and investment for large insurance portfolios," Insurance Mathematics and Economics, vol. 42, no. 1, pp. 434-444, 2008.

[5] L. Bai and J. Guo, "Optimal proportional reinsurance and investment with multiple risky assets and no-shorting constraint," Insurance Mathematics and Economics, vol. 42, no. 3, pp. 968-975, 2008.

[6] S. D. Promislow and V. R. Young, "Minimizing the probability of ruin when claims follow Brownian motion with drift," North American Actuarial Journal, vol. 9, no. 3, pp. 109-128, 2005.

[7] Z. Liang and J. Guo, "Optimal proportional reinsurance and ruin probability," Stochastic Models, vol. 23, no. 2, pp. 333-350, 2007.

[8] S. Asmussen, B. Højgaard, and M. Taksar, "Optimal risk control and dividend distribution policies: example of excess-of-loss reinsurance for an insurance for an insurance corporation," Finance and Stochastics, vol. 4, pp. 299-324, 2000.

[9] X. Zhang, M. Zhou, and J. Guo, "Optimal combinational quotashare and excess-of-loss reinsurance policies in a dynamic setting," Applied Stochastic Models in Business and Industry, vol. 23, no. 1, pp. 63-71, 2007.

[10] H. Meng and X. Zhang, "Optimal risk control for the excess of loss reinsurance policies," ASTIN Bulletin, vol. 40, no. 1, pp. 179197,2010

[11] L. Bai and J. Guo, "Optimal dynamic excess-of-loss reinsurance and multidimensional portfolio selection," Science ChinaMathematics, vol. 53, no. 7, pp. 1787-1804, 2010.

[12] C. Irgens and J. Paulsen, "Optimal control of risk exposure, reinsurance and investments for insurance portfolios," Insurance Mathematics and Economics, vol. 35, no. 1, pp. 21-51, 2004.

[13] G. Chacko and L. M. Viceira, "Dynamic consumption and portfolio choice with stochastic volatility in incomplete markets," The Review of Financial Studies, vol. 18, no. 4, pp. 1369-1402, 2005. 
[14] M. Schroder, "Computing the constant elasticity of variance option pricing formula," The Journal of Finance, vol. 44, no. 1, pp. 211-219, 1989.

[15] M. Gu, Y. Yang, S. Li, and J. Zhang, "Constant elasticity of variance model for proportional reinsurance and investment strategies," Insurance Mathematics and Economics, vol. 46, no. 3, pp. 580-587, 2010.

[16] J. Grandell, Aspects of Risk Theory, Springer, New York, NY, USA, 1991.

[17] I. Karatzas, "Optimization problems in the theory of continuous trading," SIAM Journal on Control and Optimization, vol. 27, no. 6, pp. 1221-1259, 1989.

[18] D. Mayers and C. W. Smith, "On the corporate demand for insurance," The Journal of Business, vol. 55, no. 2, pp. 281-296, 1982.

[19] H. Loubergé and R. Watt, "Insuring a risky investment project," Insurance Mathematics and Economics, vol. 42, no. 1, pp. 301310, 2008.

[20] H. R. Waters, "Some mathematical aspects of reinsurance," Insurance Mathematics and Economics, vol. 2, no. 1, pp. 17-26, 1983.

[21] O. Hesselager, "Some results on optimal reinsurance in terms of the adjustment coefficient," Actuarial Journal, no. 1, pp. 80-95, 1990.

[22] H. Gerber, An Introduction to Mathematical Theory, S. S. Huebners Foundation for Insurance Eduction, Philadelphia, Pa, USA, 1979.

[23] W. Fleming and H. Soner, Controlled Markov Process and Viscosity Solutions, Springer, New York, NY, USA, 2nd edition, 2005.

[24] Z. Liang, K. C. Yuen, and J. Guo, "Optimal proportional reinsurance and investment in a stock market with OrnsteinUhlenbeck process," Insurance Mathematics and Economics, vol. 49, no. 2, pp. 207-215, 2011. 


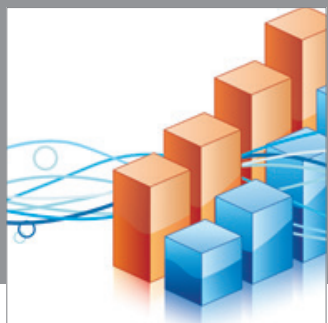

Advances in

Operations Research

mansans

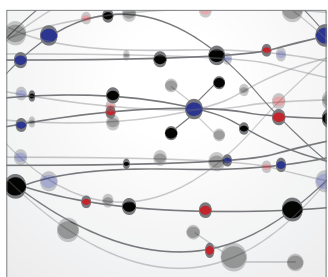

The Scientific World Journal
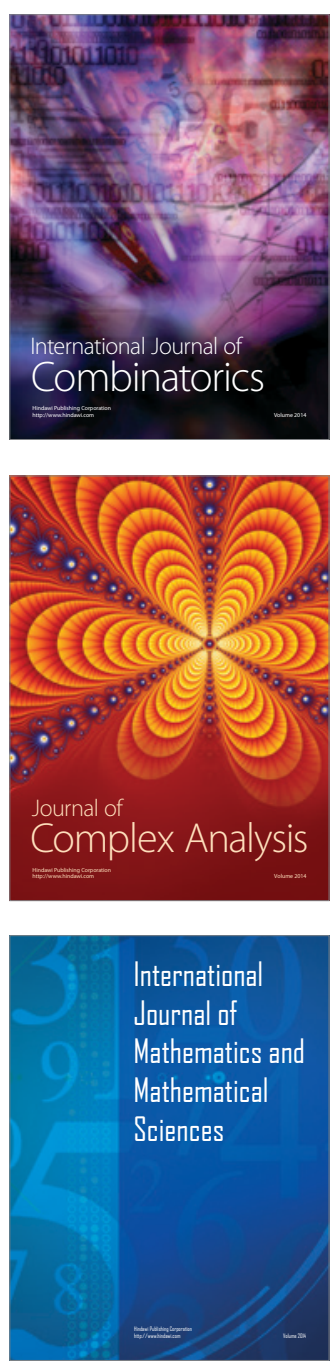
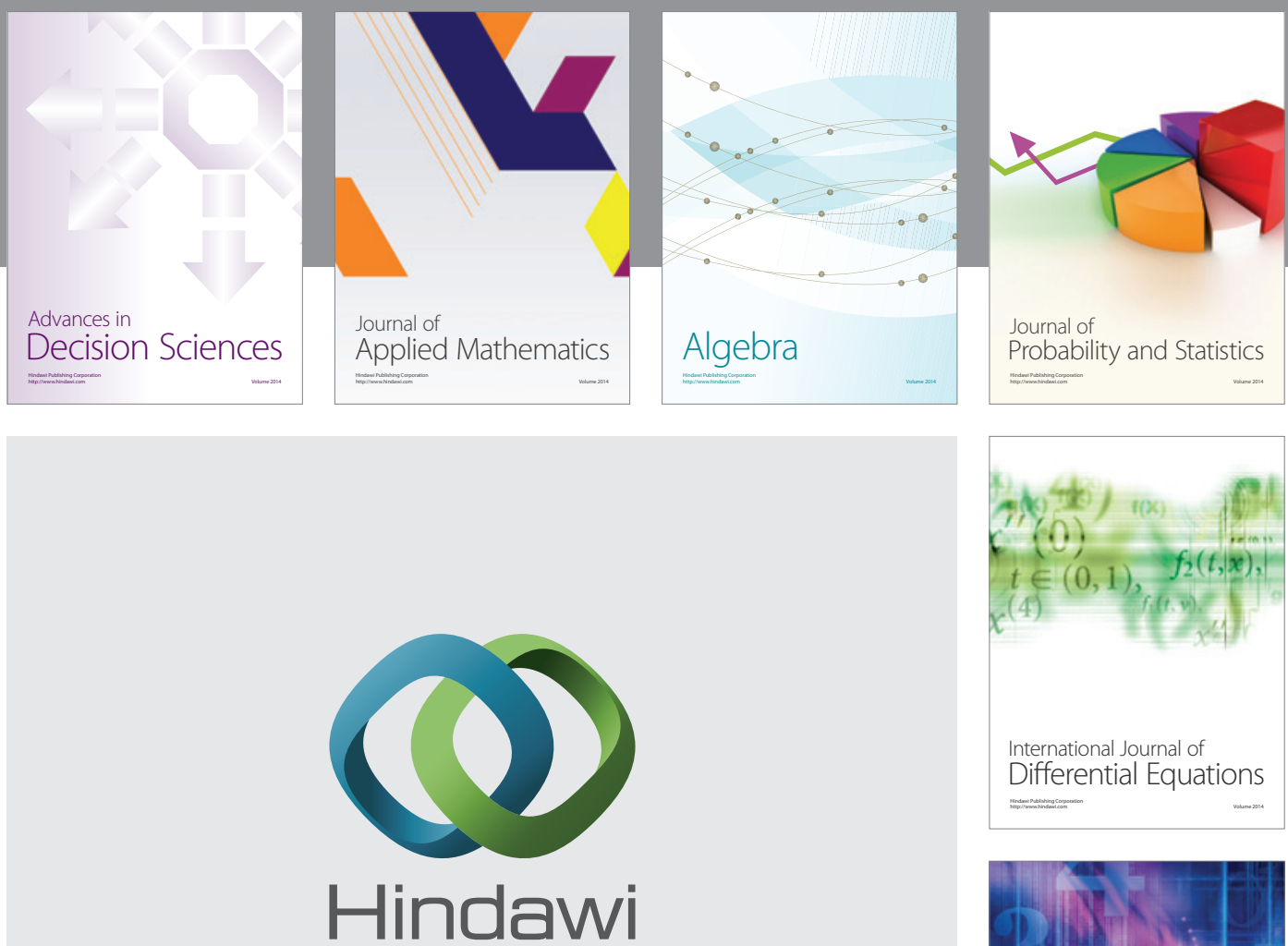

Submit your manuscripts at http://www.hindawi.com
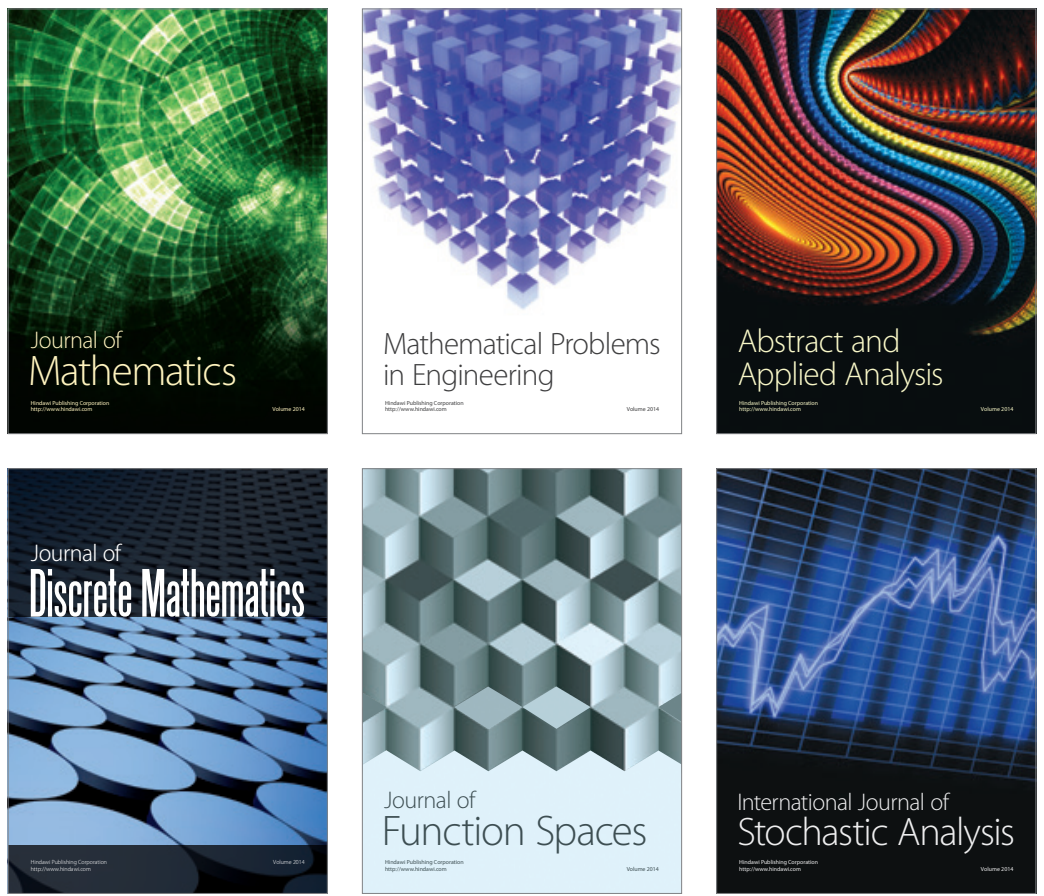

Journal of

Function Spaces

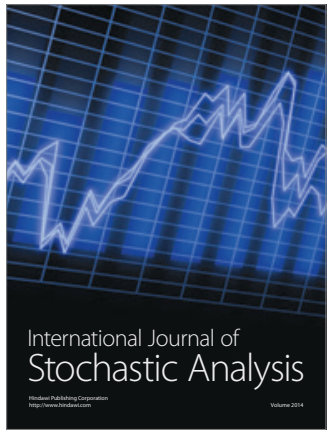

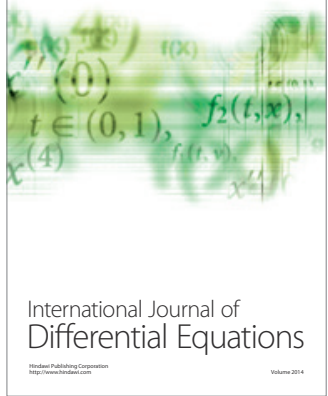
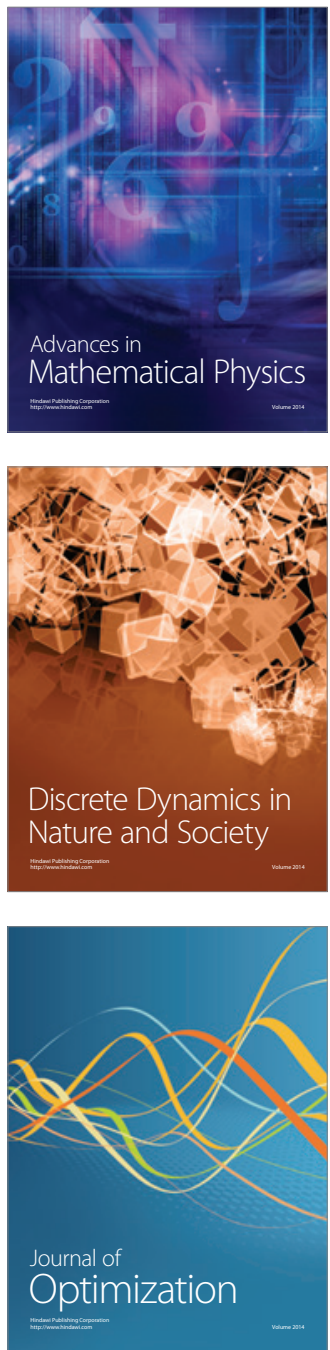